\title{
VIAS - software libre para el diseño geométrico de vías, topografía y SIG
}

\section{VIAS - freeware for road highway, topography and GIS}

\author{
John Jairo Agudelo O spina*1
}

* U niversidad Eafit, CO LO MBIA

Resumen

PAG. $52-59^{2}$

El software VIAS, de uso libre, permite realizar el diseño completo o una etapa cualquiera dentro de un proyecto de una carretera. Posee comandos fáciles de usar, además de estar en español, y suministra una información clara, de fácil manejo e interpretación además de una excelente presentación. Este se puede instalar de una manera sencilla en cualquier versión de Autocad, que es el software más usado a nivel mundial para diseño asistido por computador. Ejecuta de manera completa y precisa cada una de las etapas de un diseño de una carretera. El alineamiento horizontal se puede realizar utilizando curvas circulares o espirales. O btiene perfiles, realiza el diseño vertical, calcula y dibuja de manera automática el peralte, obtiene secciones transversales, calcula el movimiento de tierra, afectación de predios y dibuja la banca proyectada en 3 dimensiones. Además de realizar el diseño gráfico suministra una completa información en archivos de texto y en Excel que puede ser fácilmente manipulada e incorporada a cualquier informe. Adicionalmente presenta una serie de aplicaciones prácticas para topografía y SIG.

Palabras Clave: Alineamiento, peralte, perfil

Abstract

Software VIAS, free versions, allows to make the complete design or a stage anyone within a project of a highway. It has controls easy to use, is in Spanish, and provides clear information, of easy handling and interpretation in addition an excellent presentation. The software can be easily installed in any version of Autocad, the most popular CAD software in the world. It executes completely each one of the stages in a highway design . The horizontal alignment can be made using curved circular or spiral. VIAS software obtains profiles, makes the vertical design, calculates and draws automatically the superelevation, obtains cross sections, calculates the earthwork, affectation of lands and draws the road projected in 3 dimensions. Besides to make the graphical design it provides a complete information in text and Excel files that can easily be manipulated and be incorporated to any report. In addition, it presents some practical applications for survey and GIS.

Keywords: Alignment, superelevation, profile

\section{Introducción}

En los últimos años se ha incrementado la oferta de software para el diseño de vías y en la actualidad su uso se ha vuelto indispensable para poder desarrollar proyectos de manera rápida y precisa y de modo que se pueda competir en el medio en lo que respecta a calidad, costos y tiempos de ejecución. Algunos programas presentan una mayor facilidad en su manejo como en la presentación de la información obtenida, pero aún así luego de obtener los diseños se debe de dedicar un buen tiempo para adecuar dicha presentación o completar la

\footnotetext{
1 Autor de correspondencia / Corresponding author: Carrera $49 \mathrm{~N} \cong 7$ sur - 50 Medellín. Colombia. Fax: (57-4) 332 0729, E-mail: jjagudel@eafit.edu.co
}

que no está disponible.

Existe en el diseño geométrico una serie de cálculos, procedimientos y elementos requeridos en los proyectos que no se pueden obtener con ninguno de los programas existentes en el mercado. Dentro de estos elementos están el dibujo de referencias, transformación de curvas de nivel en $2 D$ a $3 D$, perfiles por una nube de puntos, etc. De igual manera el diseño del peralte algunos programas no lo ejecutan y otros lo calculan de manera errada. Los programas comerciales actuales trabajan de

\footnotetext{
2 Agradecimientos/Acknowledgements:

XIII Congreso de Ingeniería de Transporte 22 al 26 de octubre de 2007

Departamento de Ingeniería de Transportes y Logística - Escuela de Ingeniería U C Sociedad Chilena de Ingeniería de Transporte (Sochitran)
} 
dos formas. Unos tienen su propia plataforma mientras que otros utilizan como plataforma otros programas de diseño gráfico. Es claro que estos últimos presentan cierta ventaja en la medida que su plataforma sea de las más comerciales ya que todas las demás actividades diferentes al diseño mismo, como son impresión, manejo se escalas, manejo de calibres, transferencia de información, manejo de bibliotecas, etc., sean de fácil uso para el diseñador y el dibujante.

Por tal razón el programa VIAS utiliza como plataforma el software CAD más conocido y usado a nivel mundial como es el Autocad. También podrá ser utilizado por el software Intellicad cuyos alcances son similares pero que debido a su bajo costo, un $10 \%$ del costo del Autocad, su uso está tomando un gran auge en las firmas de consultoría. El programa está desarrollado en lenguajes autolisp y DCL. La mayoría de las aplicaciones o rutinas poseen cajas de dialogo de modo que su uso sea fácil y ameno y lo más importante, se puedan verificar los resultados y cálculos de modo que se cumplan todas las condiciones de tipo geométrico y normativo, antes de dibujar. Dichas cajas de dialogo cuentan además con su respectiva ayuda pero también se dispone de un manual de manejo del programa y de un instructivo de instalación para que este funcione de la manera correcta. Toda esta información, archivos y documentos podrá ser suministrada en el momento de que alguien lo requiera.

En este documento se explicarán solo los comandos principales necesarios para desarrollar cada una de las etapas dentro de un proyecto de diseño vial pero al final se presentará una lista de todas las aplicaciones del programa. El software continúa en permanente desarrollo y actualización y la idea es recibir recomendaciones y observaciones de los usuarios para su mejoramiento. (Chocontá, 2004).

\section{O bjetivos}

Para la elaboración del software VIAS, el cual se mantendrá en desarrollo permanente, se han trazado un objetivo general y otros específicos que se describen a continuación.

\section{$2.1 \mathrm{G}$ eneral}

Desarrollo de un software para diseño geométrico de vías y cálculos topográficos teniendo en cuenta las normas, recomendaciones y condiciones existentes en el medio que se quiera implementar y que sea de fácil uso tanto para el área profesional como académica.

\subsection{Específicos}

- Desarrollo de programas que permitan diseñar, calcular y dibujar el alineamiento horizontal de una vía teniendo en cuenta los diferentes tipos de curvas y de modo que se controlen las especificaciones mínimas requeridas definidas principalmente por la velocidad de diseño.

- Desarrollo de rutinas para el cálculo, diseño y dibujo de la rasante de una vía de modo que controle la visibilidad de parada y demás condiciones impuestas por la velocidad de diseño.

- Crear comandos que permitan diseñar, calcular y dibujar el peralte de una vía a partir del diseño horizontal teniendo en cuenta las especificaciones y el tipo de curva utilizado.

- Generar el movimiento de tierra de una carretera de modo que se obtenga toda la información necesaria, tanto para el presupuesto como para la construcción de la misma, a partir de los diseños horizontal, vertical y transversal.

- Desarrollar una rutina que permita obtener a partir del diseño geométrico definitivo de una carretera la información referente a la afectación predial y sus respectivas áreas.

- Crear una serie de subrutinas que permitan obtener de una manera rápida todos los datos necesarios para la elaboración de los planos apropiados para la construcción de una carretera.

- Generar los planos de Planta - Perfil de una vía, necesarios para su construcción, de una manera ágil y clara y que cumplan con los requerimientos de las entidades oficiales.

- Lograr que cada una de las subrutinas de cálculo y diseño generen los archivos de texto necesarios para la elaboración de todos los informes de un proyecto de carreteras.

- Generar rutinas que permitan realizar el replanteo de una vía de una manera rápida y precisa.

- Lograr que todas las subrutinas interactúen entre si con el fin de optimizar el manejo de la información y obtener cálculos y diseños de una manera automática.

- Desarrollar rutinas para el cálculo y dibujo de poligonales, abiertas y cerradas, de modo que se obtengan de manera automática los errores de cierre, 
ajustes y áreas de levantamiento.

- Redactar de una manera clara y un orden adecuado, el funcionamiento de cada una de las rutinas de que está compuesto el programa.

- Implementar rutinas para la importación de datos a partir de archivos de texto y de modo que se conviertan en objetos gráficos.

- Desarrollar rutinas para la exportación de datos obtenidos a partir de objetos gráficos.

- Implementar un menú propio para ser instalado en Autocad de modo que el acceso a los comandos se haga de una manera rápida.

\section{Descripción y alcances del programa}

\subsection{Definir especificaciones}

Inicialmente se deben definir las especificaciones generales del proyecto o de cada uno de los ejes con que cuenta dicho proyecto. Para esto se procede de la siguiente manera:

Comando: ESPRO (Especificaciones del Proyecto) Menú: Vias $\rightarrow$ Espro

Aparece la siguiente caja de dialogo:

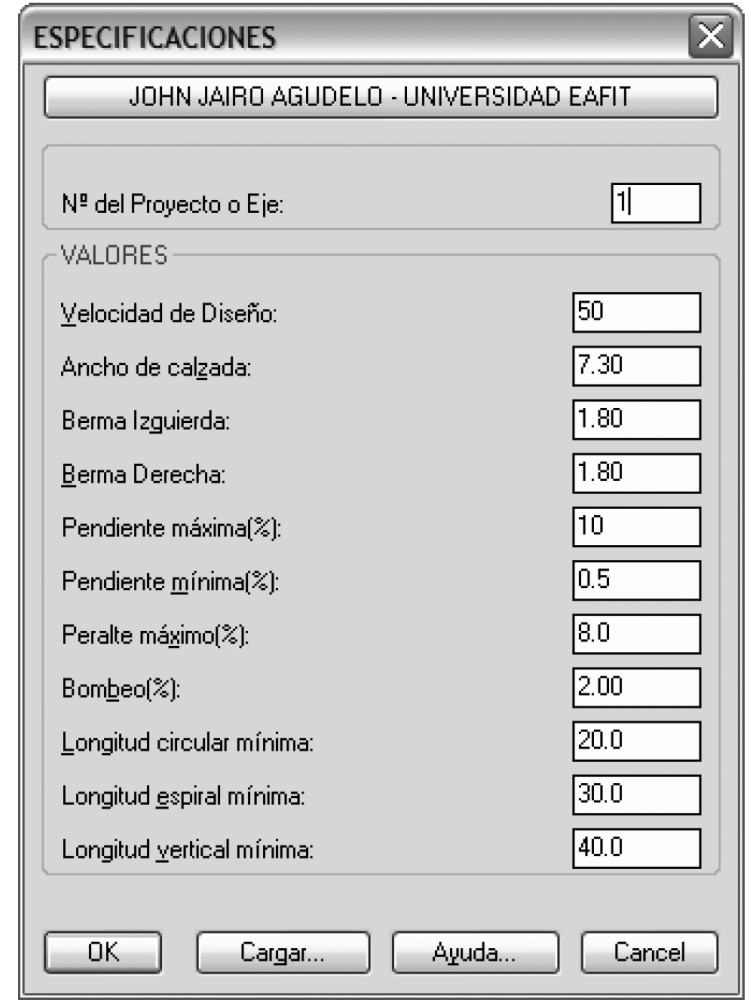

Figura 1. Caja de diálogo para definir especificaciones
Se debe de ingresar el número del eje o del proyecto y cada una de las especificaciones y finalizar con la opción $\mathrm{OK}$. Si no se define ningún eje o proyecto el programa asumirá los valores que aparecen en la caja de dialogo mostrada arriba.

\subsection{Alineamiento horizontal}

El programa cuenta con cuatro comandos para el diseño de curvas horizontales: DCC (Dibujo Curva Circular), DCEC (Dibujo Curva Espiral Circular), DCEE (Dibujo Curva Espiral Espiral) y ESPIAS (Espiral asimétrica).

Los cuatro comandos generan además de el dibujo un cuadro de elementos, un vinculo con atributos ubicado en el PI, un archivo de texto y un archivo Excel. Además es posible anular curvas ya diseñadas y reiniciar el diseño en cualquier punto.

Cada uno de estos posee su propia caja de diálogo que se presenta al señalar las líneas que conforman el PI horizontal a diseñar. U na de estas es la siguiente:

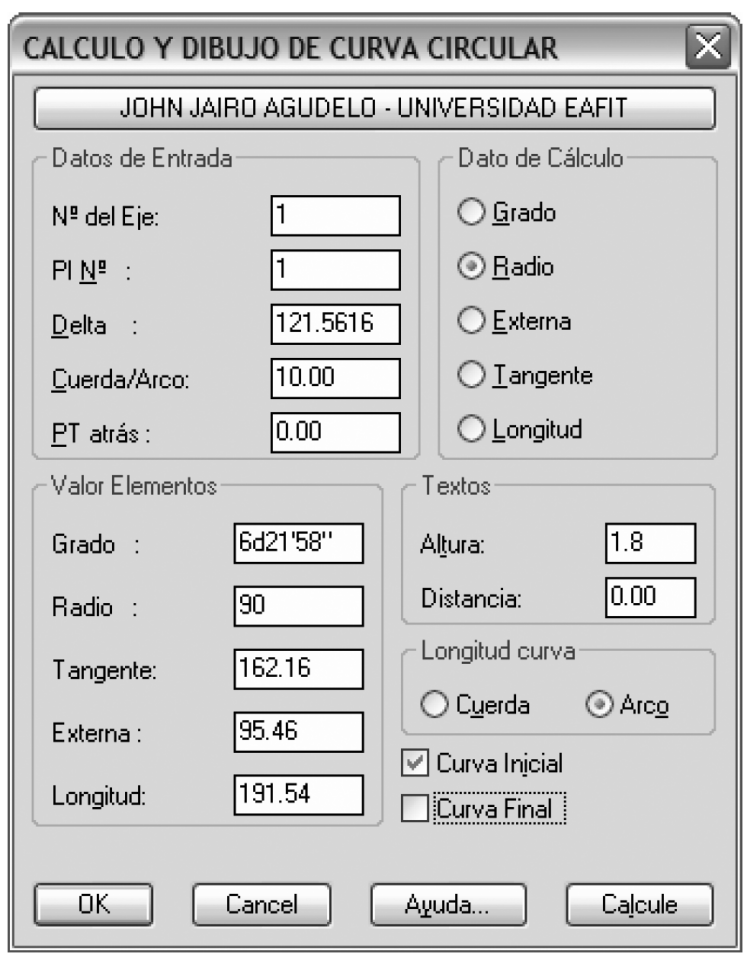

Figura 2. Caja de diálogo para comando DCC 


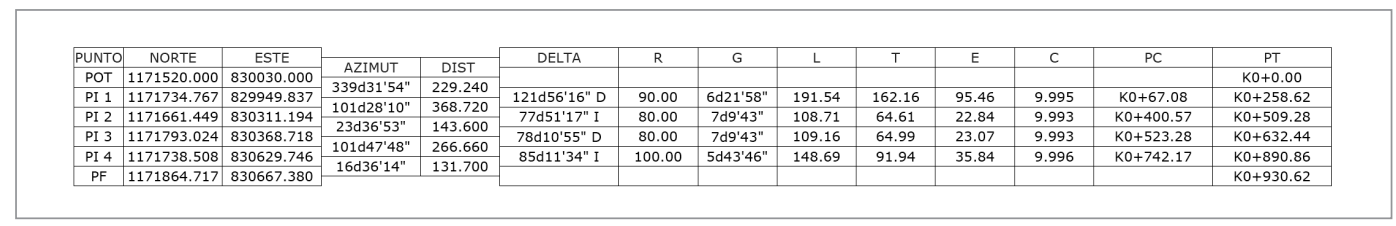

Figura 3. Cuadro de elementos generado con comando DCC

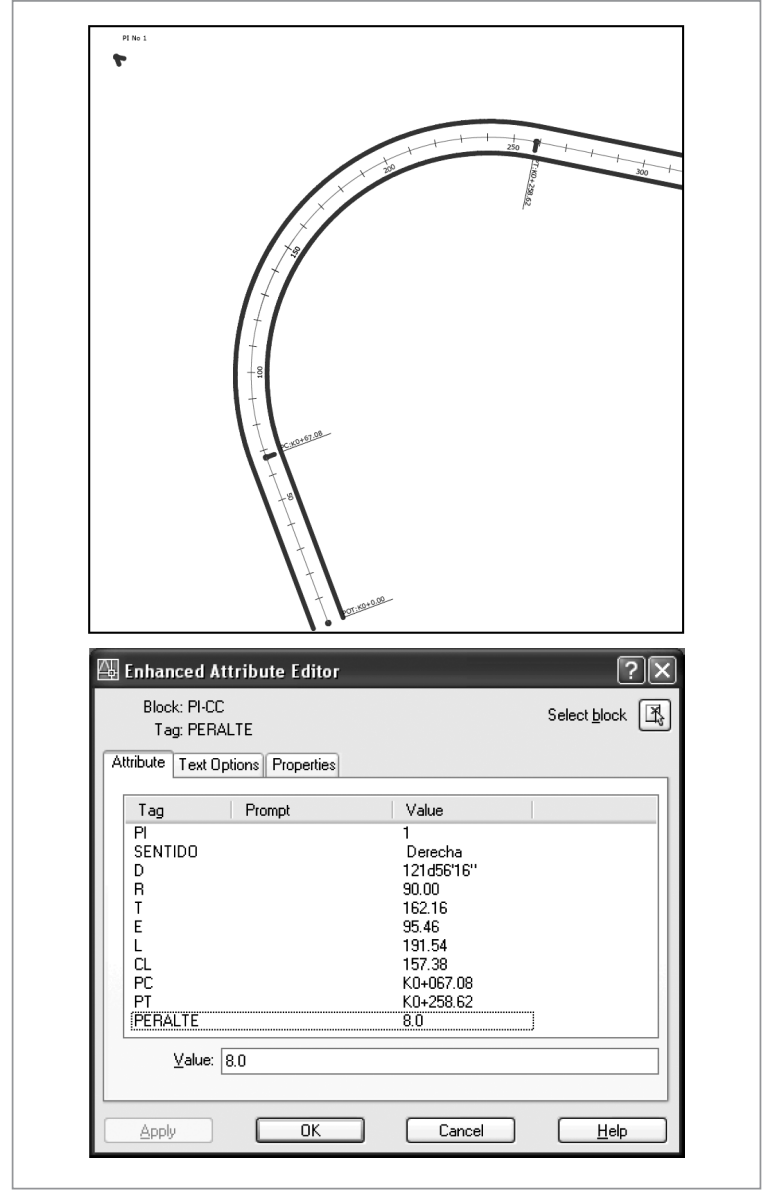

Figuras 4 y 5 . Dibujo de curva y cuadro de atributos generado con comando DCC

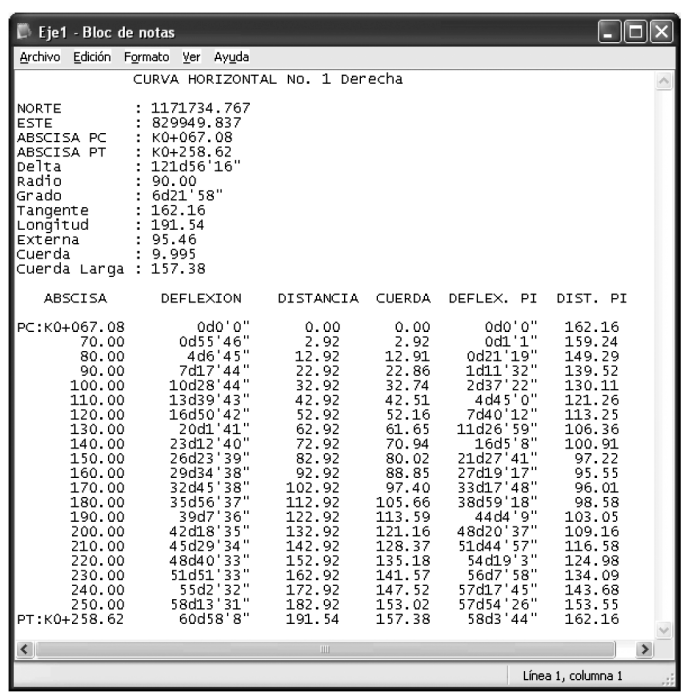

Figura 6. Archivo de texto generado con comando DCC

\subsection{O btención de perfiles longitudinales}

El programa cuenta con cuatro comandos para obtener perfiles longitudinales a lo largo de un eje. Desde una topografía con el comando PERFIL, desde una malla de puntos con el comando PERMAL, desde una nube de puntos con el comando PERPUN y desde un archivo con el comando IP.

Cuando se trata del eje de una vía se utiliza el comando PERFIL el cual se describe a continuación:

Comando: PERFIL

Menú: Vias $\rightarrow$ Perfil $\rightarrow$ Eje

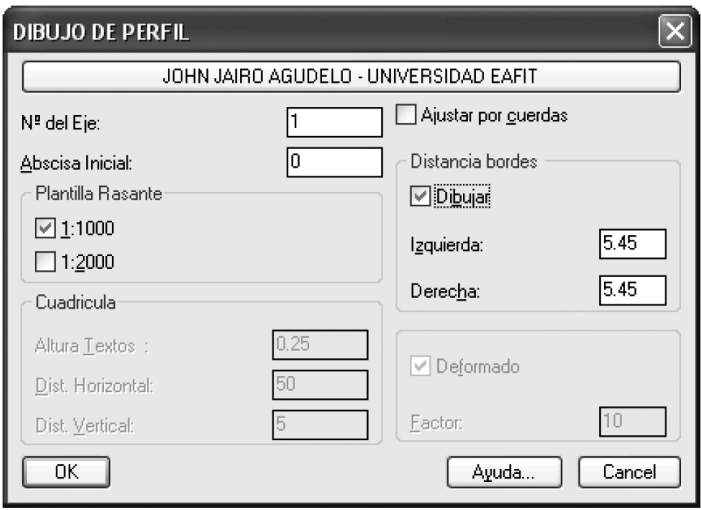

Figura 7. Caja de diálogo de comando PERFIL

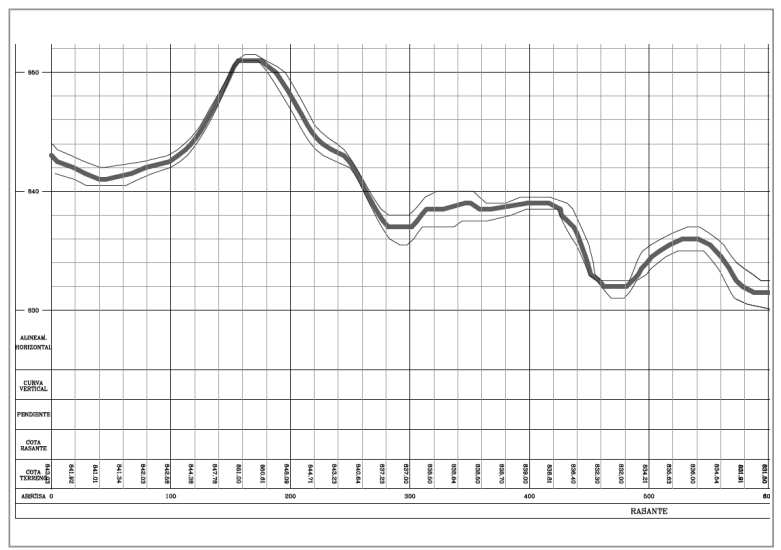

Figura 8. Perfil longitudinal generado con comando PERFIL

\subsection{Alineamiento vertical}

Se cuenta con varias herramientas para el buen diseño de la rasante. U na de ellas es la que permite el cálculo y dibujo de curvas verticales. 
Comando: CCV1

Menú: Vias $\rightarrow$ Alin. Vertical $\rightarrow$ CV - 1000

Al señalar las líneas que conforman el PIV a diseñar se presenta la siguiente caja de diálogo:

\begin{tabular}{|c|c|c|c|}
\hline \multicolumn{4}{|c|}{ DIBUJO DE CURVA VERTICAL } \\
\hline \multicolumn{4}{|c|}{ JOHN JAIROA AGUDELO - UNIVERSIDAAD EAFIT } \\
\hline NNo del Eje: & 1 & Absc. PV anterior: & 0 \\
\hline Curva Numero: & 1 & Cota Minima Datos: & 820.00 \\
\hline Pend. Inicial (\%): & -4.90 & Altura Iextos: & 0.18 \\
\hline Pend. Final (\%): & 4.50 & $\square$ Curva Final & \\
\hline Long. Inicial: & 30 & $\square$ Calcular con Externa & \\
\hline Long. Final: & 30 & Externa: & \\
\hline \multicolumn{4}{|c|}{ NO CUMPLE VISIBILIDAD DE PABAADA - L min=94 } \\
\hline \multicolumn{4}{|c|}{ DEBE MODIFICAR SISTEMA DE COORDENADAS } \\
\hline OK & 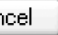 & Chegueo... & Ayuda. \\
\hline
\end{tabular}

Figura 9. Caja de diálogo para diseño de curvas verticales

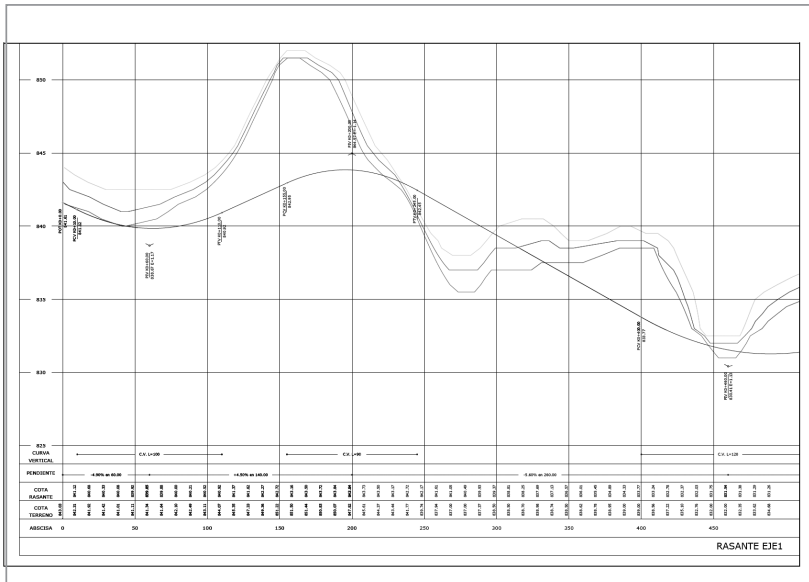

Figura 10. Diseño de curvas verticales

El comando también genera cuadro de atributos y un archivo de texto con los resultados. Al igual que las curvas horizontales estas también se pueden anular.

\subsection{Cálculo y diseño del peralte}

El programa cuenta con tres comandos para el cálculo del peralte. U no para el cálculo del peralte de curvas circulares, otro para curvas espiralizadas y un tercero para el dibujo del diagrama.

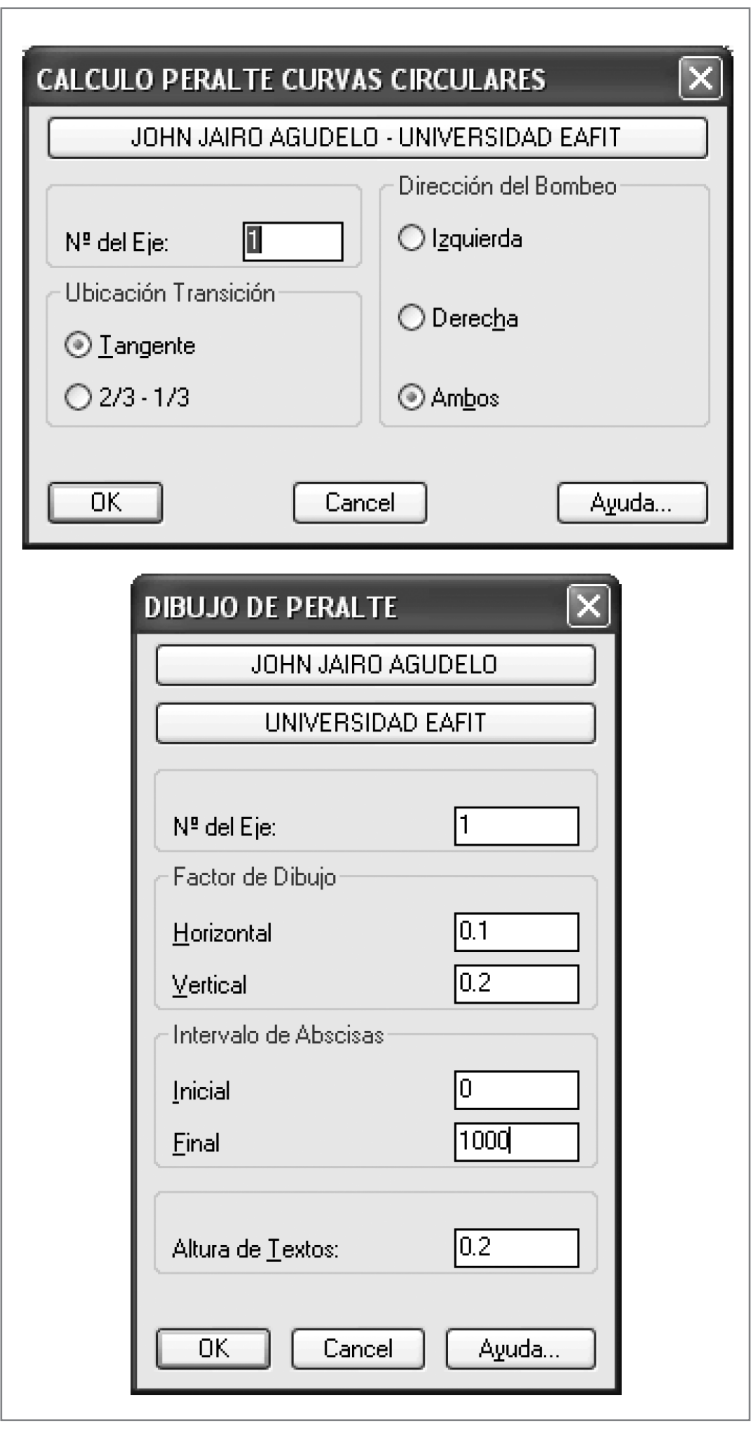

Figura 11. Cajas de diálogo para el cálculo y dibujo del peralte El archivo con el cálculo es el siguiente:

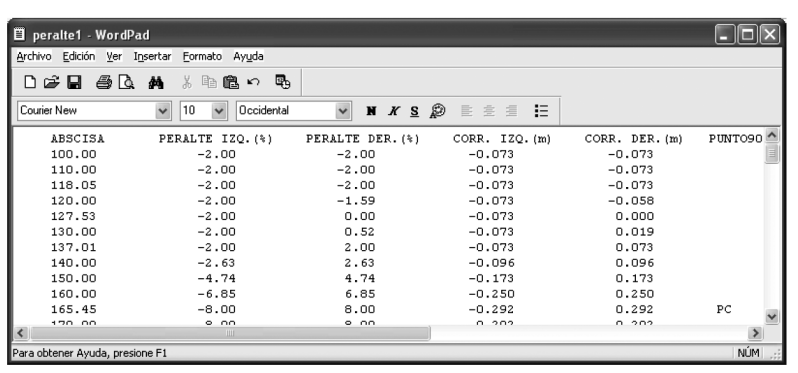

Figura 12 Archivo generado por comando PERACIR

Parte de un diagrama de peralte dibujado se muestra a continuación: 


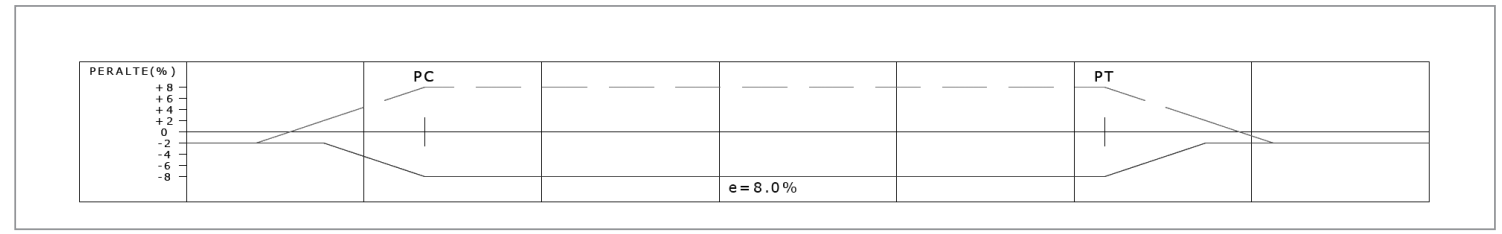

Figura 13. Diagrama de peralte dibujado

3.6 Movimiento de tierra

Para el cálculo de movimiento de tierra se tienen varios comandos, de los cuales se presentan los más importantes.

Comando : TOMASE

Menú : Vías $\rightarrow$ Secciones Transversales $\rightarrow$ Tomar secciones

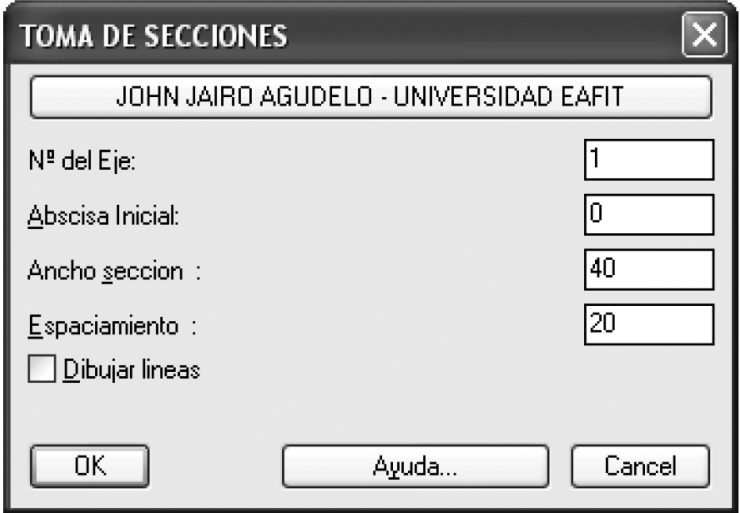

Figura 14. Caja de diálogo de comando TO MASE

Comando : RASTO

Menú $\quad$ Vias $\rightarrow$ Secciones Transversales $\rightarrow$ Cuadro de Banca

El comando genera de manera simultánea un cuadro de datos en Autocad, en Excel y en un archivo de texto. Al llamar el comando se presenta la siguiente caja de diálogo:

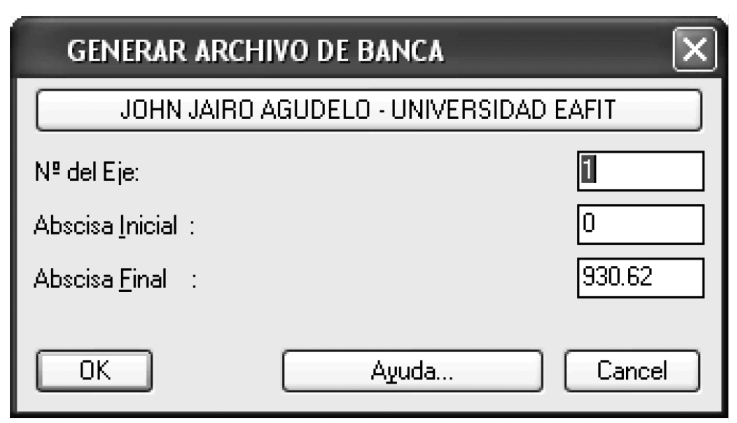

Figura 15. Caja de diálogo de comando RASTO
El archivo generado en Excel presenta el siguiente formato:

Tabla 1. Generada en Excel con comando RASTO

\begin{tabular}{||c|c|c|c|c|c|c||}
\hline ABSCISA & $\begin{array}{c}\text { COTA } \\
\text { TERRENO }\end{array}$ & $\begin{array}{c}\text { COTA } \\
\text { RASANTE }\end{array}$ & $\begin{array}{c}\text { PERALTE } \\
\text { IZQ (\%) }\end{array}$ & $\begin{array}{c}\text { PERALTE } \\
\text { DER (\%) }\end{array}$ & $\begin{array}{c}\text { COTA } \\
\text { BORDE } \\
\text { IZQ }\end{array}$ & $\begin{array}{c}\text { COTA } \\
\text { BORDE } \\
\text { DER }\end{array}$ \\
\hline 0.00 & 843.03 & 842.100 & -2.00 & -2.00 & 842.027 & 842.027 \\
\hline 10.00 & 842.31 & 841.659 & -2.00 & -2.00 & 841.586 & 841.586 \\
\hline 20.00 & 841.92 & 841.336 & -1.93 & -2.00 & 841.265 & 841.263 \\
\hline 30.00 & 841.42 & 841.130 & 0.18 & -2.00 & 841.137 & 841.057 \\
\hline 40.00 & 841.01 & 841.043 & 2.29 & -2.29 & 841.126 & 840.959 \\
\hline
\end{tabular}

\section{Comando : SECTO}

Menú $\quad$ : Vías $\rightarrow$ Secciones Transversales $\rightarrow$ Banca

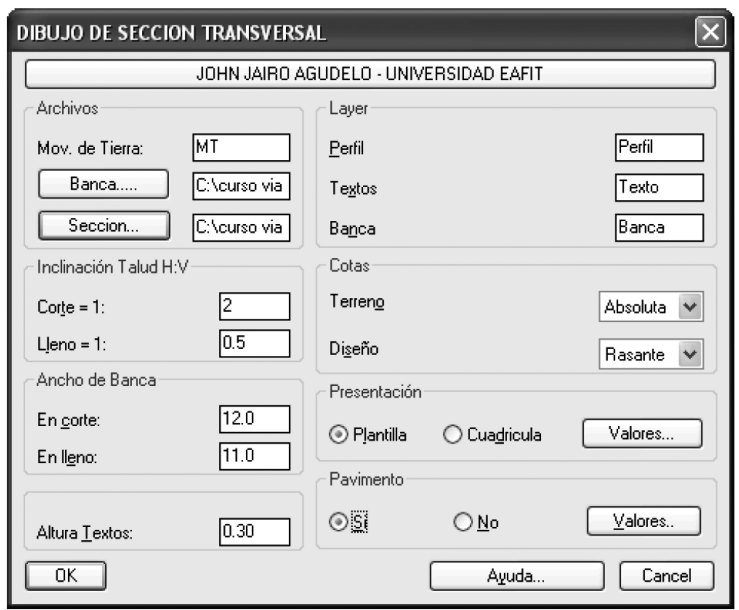

Figura 16. Caja de diálogo de comando SECTO

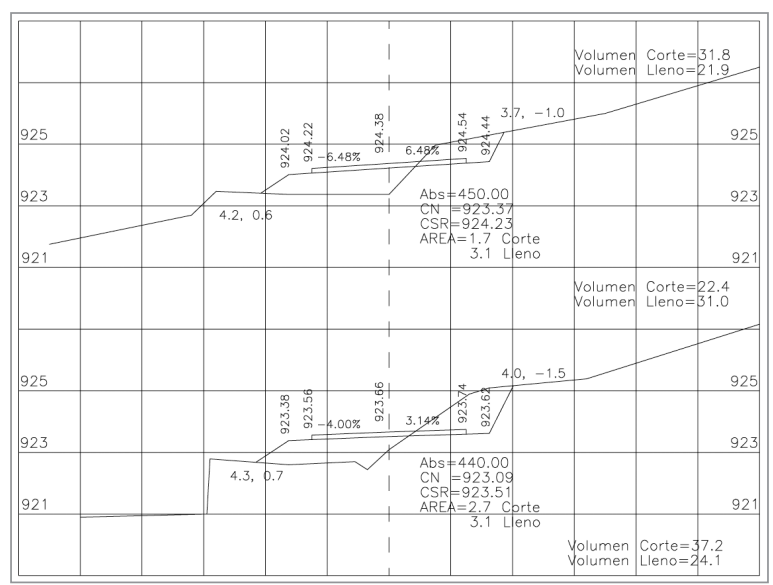

Figura 17. Secciones obtenidas con comando RASTO 


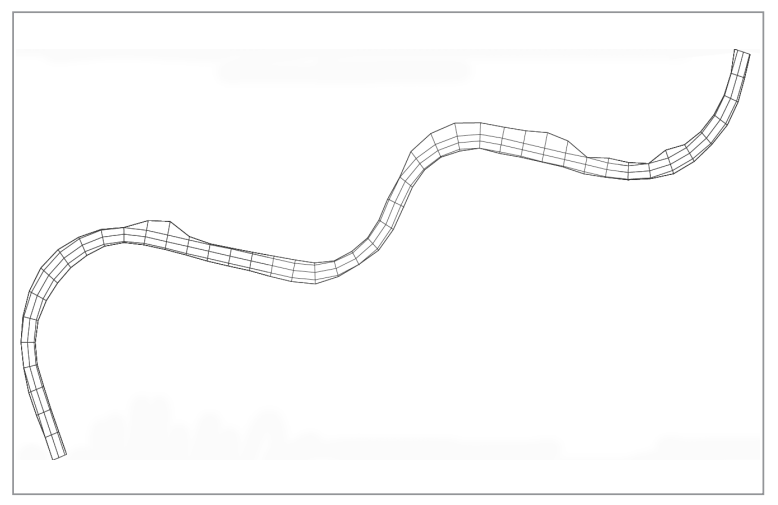

Figura 18. U bicación de chaflanes en planta

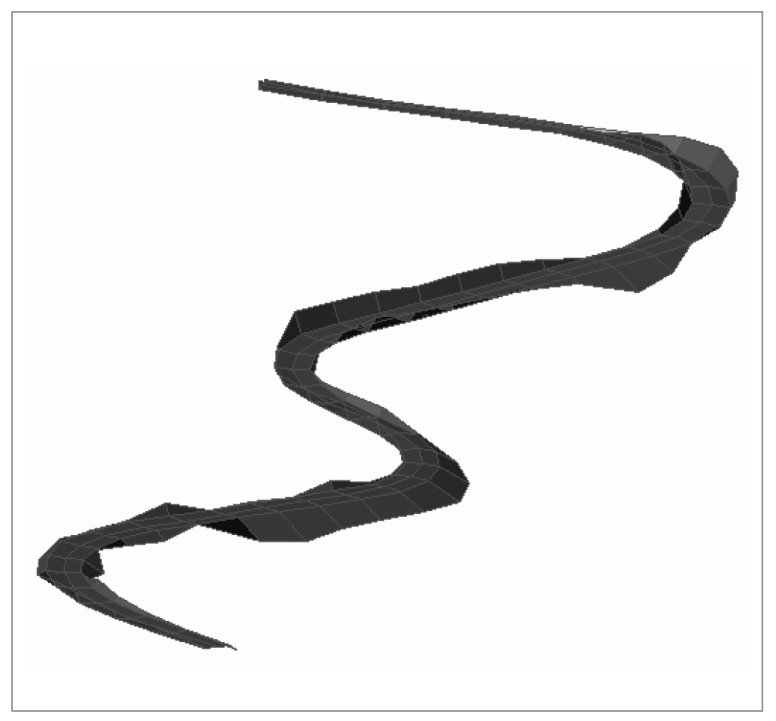

Figura 19. Vía tridimensional obtenida

\subsection{Presentación en plantillas}

Junto con el programa se suministran además las plantillas para que el proyecto pueda tener una excelente presentación. Las plantillas que se suministran son de Planta - Perfil y secciones transversales. Estas plantillas pueden ser modificadas a gusto del usuario para diferentes escalas o también pueden ser creadas con las especificaciones que se consideren convenientes ya que el programa permite adaptarse a cualquier plantilla.

A continuación se muestra una imagen de una plantilla de planta - perfil donde se ha incorporado el diseño horizontal, vertical y de peralte de un proyecto realizado con el programa. Se puede observar en el plano: Alineamiento horizontal, cuadro de elementos, escala gráfica, especificaciones, peralte, diseño vertical.

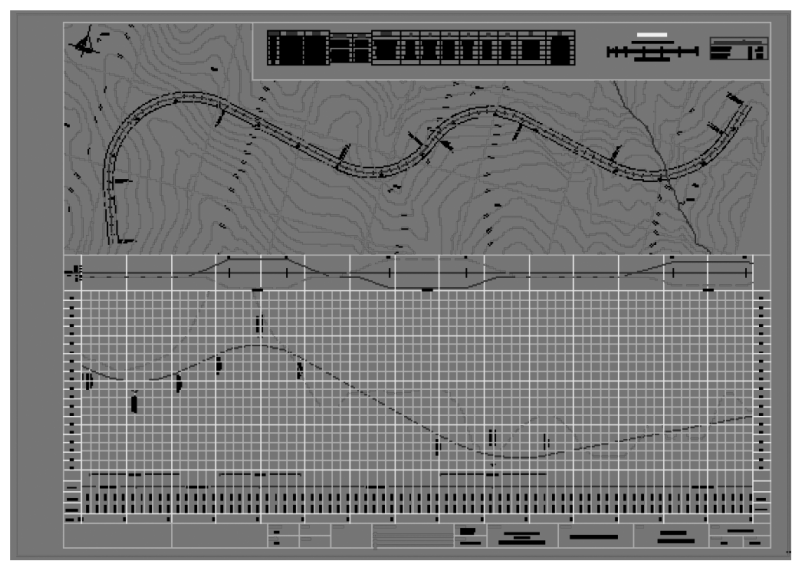

Figura 20. Plano de Planta - Perfil

\subsection{0 tros comandos}

En este documento se han incorporado solo algunas de las rutinas más importantes para el diseño de vías. Por lo tanto a continuación se hace un listado de otras aplicaciones o comandos con que cuenta el programa.

- Obtención de perfiles de una nube de puntos o archivo

- Ajuste y control automático del diseño de rasante

- Obtención y dibujo de secciones transversales

- Dibujo de referencias, Cálculo de áreas y Replanteo de puntos

- Cálculo y dibujo de levantamientos topográficos

- Control de cierre, cálculo de precisión y ajuste de poligonales

- Importación y exportación de puntos y Exportación de datos de una poligonal

- Obtención automática de datos de parcelas

- Transformación de coordenadas, Cálculo de azimut

- Dibujo de cuadrícula de coordenadas

- Abscisado y exportación de coordenadas de un eje

- Interpolación de puntos tridimensionales

- Asignación de elevación a curvas de nivel y Colocación automática de cotas

- O btención automática de cantidad, longitud y área de elementos Iongitudinales.

- Triangulación de puntos tridimensionales. 


\section{Conclusiones}

- El programa es de uso libre y se puede adaptar a cualquier manual o norma con modificaciones fáciles y rápidas. Está diseñado en lenguajes Autolisp y $\mathrm{DCL}$

- La gran mayoría de comandos posee caja de diálogo de modo que su uso sea más fácil y amigable.

- Además de tener un manual donde se describe el funcionamiento de cada comando de manera detallada, cada caja de diálogo posee su propia Ayuda.

- Se puede usar de manera integrada o en cualquier etapa del diseño de una vía.

\section{Referencias}

Chocontá Rojas, P.A. (2004), Libro "Diseño Geométrico de Vías), Escuela Colombiana de Ingeniería - Edición abril de 2004, ISBN 958-8060-39-7 